\title{
BENDING, BUCKLING, AND FORCED VIBRATION ANALYSES OF NONLOCAL NANOCOMPOSITE MICROPLATE USING TSDT CONSIDERING MEE PROPERTIES DEPENDENT TO VARIOUS VOLUME FRACTIONS OF $\mathrm{CoFe}_{2} \mathrm{O}_{4}-\mathrm{BaTiO}_{3}$
}

\author{
Mehdi Mohammadimehr, Rasoul Rostami \\ University of Kashan, Department of Solid Mechanics, Faculty of Mechanical Engineering, Kashan, Iran \\ e-mail: mmohammadimehr@kashanu.ac.ir; r_rostami@grad.kashanu.ac.ir
}

\begin{abstract}
In this article, the bending, buckling, free and forced vibration behavior of a nonlocal nanocomposite microplate using the third order shear deformation theory (TSDT) is presented. The magneto-electro-elastic (MEE) properties are dependent on various volume fractions of $\mathrm{CoFe}_{2} \mathrm{O}_{4}-\mathrm{BaTiO}_{3}$. According to Maxwell's equations and Hamilton's principle, the governing differential equations are derived. These equations are discretized by using Navier's method for an MEE nanocomposite Reddy plate. The numerical results show the influences of elastic foundation parameters such as aspect ratio, length to thickness ratio, electric and magnetic fields and various volume fractions of $\mathrm{CoFe}_{2} \mathrm{O}_{4}-\mathrm{BaTiO}_{3}$ on deflection, critical buckling load and natural frequency. The natural frequency and critical buckling load increases with the increasing volume fraction of $\mathrm{CoFe}_{2} \mathrm{O}_{4}-\mathrm{BaTiO}_{3}$, also the amplitude vibration decreases with an increase in the volume fraction. This model can be used for various nanocomposite structures. Also, a series of new experiments are recommended for future work.
\end{abstract}

Keywords: bending and buckling analysis, free and forced vibration analysis, nonlocal nanocomposite microplate, various volume fractions of $\mathrm{CoFe}_{2} \mathrm{O}_{4}-\mathrm{BaTiO}_{3}$

\section{Introduction}

In the recent years, the use of nano-technology is a subject of the main discussion in the world of engineering sciences. Nano-technology is science in which the design and application of nanostructures relates different properties at the nanoscale. The size of nanoparticles and their dispersion in a matrix composite is one of the ways to achieve desired properties of nanocomposites. According to the nanometer-scale, the reinforcement particles in nanocomposites, intermolecular forces between the matrix and reinforcing is much greater than in ordinary composites, which improves properties of the nanocomposites. The reinforcing phase in terms of the material can be used as polymeric, metal and ceramic, which, according to different properties of each, have different applications. Because of their magnetoelectric coupling effects, magnetoelectric-elastic (MEE) materials have been widely employed in many technological fields, such as sensor and actuator applications, robotics, medical instruments, structural health monitoring, energy harvesting. Many researchers have carried out static, buckling, and free vibration analysis of nanocomposites, see Sih and $\mathrm{Yu}$ (2005) who analyzed the volume fraction effect of a MEE composite on enhancement and impediment of crack growth. Their results showed that with the increasing electric field to normal stress ratio and the volume fraction effect of the MEE composite, the crack growth increased and decreased, respectively. Ke and Wang (2014) examined free vibration of size-dependent magneto-electro-elastic nanobeams based on the nonlocal elasticity theory. By using the Hamilton principle, the governing equations and boundary conditions were derived and discretized by using the differential quadrature method (DQM) to determine natural frequencies. Their results showed that with the increasing magnetic and electric potential, the 
natural frequencies of nanobeams increased. Shokrani et al. (2016) employed the generalized differential quadrature method (GDQM) to the buckling analysis of double orthotropic nanoplates (DONP) embedded in elastic media under biaxial, uniaxial and shear loadings. Their results showed that for higher values of the non-local parameter, the shear buckling was not dependent on the van der Waals and Winkler moduli. Lang and Xuewu (2013) studied the buckling and vibration of functionally graded magneto-electro-thermo-elastic circular cylindrical shells. Based on using the third order shear theory (TSDT), they employed Hamilton's principle to obtain equations of motion and numerical solutions to find the natural frequencies. Ghorbanpour Arani et al. (2012) investigated the effect of the CNT volume fraction on the magneto-thermoelectro-mechanical behavior of a smart nanocomposite cylinder. Their results indicated that the influence of internal pressure on the radial stress was larger than thermal, magnetic and electric fields. Also, their results are very useful for the optimization of nano-composite structures. Xin and $\mathrm{Hu}$ (2015) analyzed free vibration of multilayered magneto-electro-elastic plates based on the state space approach (SSA) and the discrete singular convolution (DSC) algorithm. The results showed that the piezoelectric effect had a tendency to increase the stiffness of the plate, and vice versa for the magnetostrictive effect. Karimi et al. (2015a) investigated surface effects and non-local two variable refined plate theories that were combined on the shear/biaxial buckling and vibration of rectangular nanoplates. Their results showed that by increasing the non-local parameter, the effects of surface on the buckling and vibration increased. Shooshtari and Razavi (2015) studied nonlinear free vibration behavior of a symmetrically laminated MEE doubly-curved thin shell resting on an elastic foundation. By introducing a force function and using the Galerkin method, the nonlinear partial differential equations of motion were reduced to a single nonlinear ordinary differential equation. That equation was solved analytically by the Lindstedt-Poincaré perturbation method. Their results showed that the shear constant coefficient of the foundation had much greater effect on the natural frequency when compared with the spring constant coefficient, and both of those coefficients increased the fundamental natural frequency. Ebrahimi and Nasirzadeh (2016) analyzed free vibration of thick nanobeams based on Eringen nonlocal elasticity theory and Timoshenko beam theory. Chen et al. (2014) studied free vibration of multilayered MEE plates under combined clamped/free lateral boundary conditions. Using semi-analytical solution, they obtained the natural frequency. Their results illustrated the effect of stacking sequences and magneto-electric coupling on natural frequencies and mode shapes. Karimi et al. (2015c) analyzed size-dependent free vibration characteristics of rectangular nanoplates considering surface stress effects. Numerical results demonstrated that the obtained natural frequency by considering the surface effects was lower than that without considering the surface properties. Razavi and Shooshtari (2015) employed nonlinear free vibration of symmetric MEE laminated rectangular plates with simply supported boundary conditions. Their results for the nonlinear natural frequency ratio were compared with the available results for isotropic, laminated layers and piezo-layers and laminated MEE plates. Their results depicted that the foundation parameters, negative electric potential and positive magnetic potential increased the equivalent stiffness of the system. Using Bert's model, Khan et al. (2014) studied free and forced vibration characteristics of bimodular composite laminated circular cylindrical shells. The results indicated that the relative difference of positive and negative half cycle frequencies was considerably less for single layer orthotropic shells, and it was significant for cross-ply shells with the axisymmetric mode of vibration. Du et al. (2014) illustrated nonlinear forced vibration analysis of infinitely long functionally graded cylindrical shells using the Lagrangian theory and the multiple scale method. Their results found that the power-law exponent had not any influence on the qualitative behavior of FG cylindrical shells, but it would change the amplitude in a complex nonlinear way. Hasani Baferani et al. (2011) presented free vibration analysis of FG thick rectangular plates resting on an elastic foundation. They obtained governing equations of motion using the third order shear deformation plate theory and Hamilton's 
principle. Their results showed that the Pasternak elastic foundation drastically changed the natural frequency. Also some boundary conditions and in-plane displacements had significant effects on the natural frequency of FG thick plates. Arefi (2015) analyzed free vibration of a FG solid and annular circular plates with two functionally graded piezoelectric layers at the top and bottom subjected to an electric field. Sobhy (2013) investigated buckling and free vibration of exponentially graded sandwich plates resting on elastic foundations under various boundary conditions. The governing equations of plates were derived by using various shear deformation plate theories. They showed influence of the inhomogeneity parameter, aspect ratio, thickness ratio and foundation parameters on natural frequencies and critical buckling loads. Zidour et al. (2014) illustrated buckling of chiral single-walled carbon nanotubes by using the nonlocal Timoshenko beam theory. Their results showed influence of a nonlocal small-scale coefficient and the vibration mode number on the nonlocal critical buckling loads. Karimi et al. (2015b) studied influence of the nonlocal parameter, van der Waals, Winkler, shear modulus on shear vibration and buckling of double-layer orthotropic nanoplates resting on an elastic foundation.

In this article, bending, buckling, free and forced vibration of a magneto-electro-elastic (MEE) microplate based on the third order shear deformation theory (TSDT) is presented. According to Maxwell's equations and Hamilton's principle, the governing differential equation is obtained. These equations discretized by using Navier's method for a MEE microplate with all edges simply supported boundary enabled determination of the deflection, critical buckling load, natural frequency, response of the system as well as the electric and magnetic intensity of the microplate. The numerical results show the influence of elastic foundation parameters, aspect ratio $l / b$, length to thickness ratio $l / h$, volume fraction, normal pressure on the deflection, critical buckling load, natural frequency, response of the system and the electric and magnetic intensity.

\section{Nonlocal theory of the MEE}

The non-local modulus of elasticity was presented by Eringen (1983). This model states that the stress of a point in the micro and nano dimension is dependent on the strain in all parts of the model. The fundamental equations of a homogeneous and isotropic non-local elastic solid are given by Eringen (2002)

$$
\sigma_{i j}^{n l}(x)=\int_{V} \alpha\left(\left|x-x^{\prime}\right|, \tau\right) \sigma_{i j}^{\prime} d V\left(x^{\prime}\right) \quad \forall x \in V
$$

For the MEE solid, the nonlocal fundamental equations for magnetic induction and electric displacement can be obtained as follows

$$
\begin{array}{ll}
D_{i j}^{n l}(x)=\int_{V} \alpha\left(\left|x-x^{\prime}\right|, \tau\right) D_{i j}^{\prime} d V\left(x^{\prime}\right) & \forall x \in V \\
B_{i j}^{n l}(x)=\int_{V} \alpha\left(\left|x-x^{\prime}\right|, \tau\right) B_{i j}^{\prime} d V\left(x^{\prime}\right) & \forall x \in V
\end{array}
$$

where $\sigma_{i j}^{n l}, \sigma_{i j}^{\prime}, D_{i j}^{n l}, D_{i j}^{\prime}, B_{i j}^{n l}$ and $B_{i j}^{\prime}$ are the nonlocal and local stress tensor, components of the nonlocal and local electric displacements, components of the nonlocal and local magnetic inductions, respectively. $\alpha\left(\left|x-x^{\prime}\right|, \tau\right)$ is the nonlocal modulus, $\left|x-x^{\prime}\right|$ is the Euclidean distance, $\tau=e_{0} a / l$ is defined as the small scale parameter.

According to Eringen $(1983,2002)$, the nonlocal elasticity theory can be simplified to partial differential equations. Thus we have

$$
\left[1-\left(e_{0} a\right)^{2} \nabla^{2}\right] \sigma_{i j}^{n l}=\sigma_{i j}^{\prime} \quad\left[1-\left(e_{0} a\right)^{2} \nabla^{2}\right] D_{i j}^{n l}=D_{i j}^{\prime} \quad\left[1-\left(e_{0} a\right)^{2} \nabla^{2}\right] B_{i j}^{n l}=B_{i j}^{\prime}
$$




\section{Constitutive equations of the MEE nanocomposite microplate}

Consider an MEE nanocomposite microplate with length $l$, width $b$ and thickness $h$, resting on an elastic foundation as shown in Fig. 1. A Cartesian coordinate system $(x, y, z)$ is considered such that the $z$ direction denotes thickness of the nanocomposite microplate.

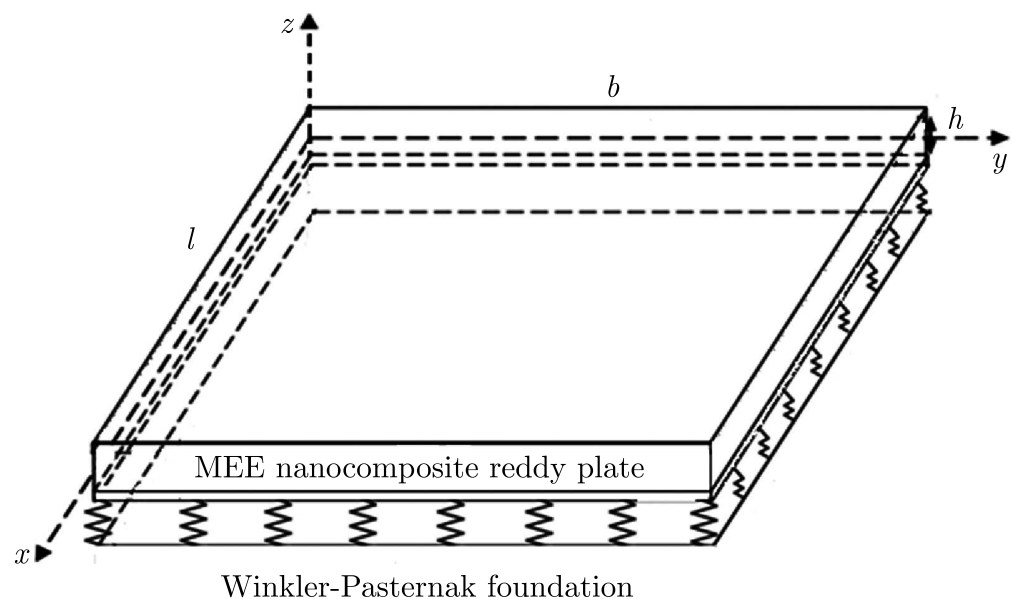

Fig. 1. Schematic of an MEE nanocomposite microplate on the elastic foundation

Based on the third-order shear deformation theory (TSDT) for a nanocomposite plate, the displacements of an arbitrary point in the beam along the $x, y$ and $z$ axes are denoted by $u_{1}(x, y, z, t), u_{2}(x, y, z, t)$ and $u_{3}(x, y, z, t)$, respectively. They are written as follows

$$
\begin{aligned}
& u_{1}(x, y, z, t)=u(x, y, t)+z\left[\psi_{x}(x, y, t)-\frac{4}{3}\left(\frac{z}{h}\right)^{2}\left[\psi_{x}(x, y, t)+w(x, y, t)_{, x}\right]\right] \\
& u_{2}(x, y, z, t)=v(x, y, t)+z\left[\psi_{y}(x, y, t)-\frac{4}{3}\left(\frac{z}{h}\right)^{2}\left[\psi_{y}(x, y, t)+w(x, y, t)_{, y}\right]\right] \\
& u_{3}(x, y, z, t)=w(x, y, t)
\end{aligned}
$$

where $u, v, w$ are the mid-plane displacements of the MEE rectangular nanocomposite microplate along the $(x, y, z)$ coordinate directions, respectively, $\psi_{x}, \psi_{y}$ denote rotations of the plate crosssection and $t$ is time.

The linear constitutive equations for the MEE nanocomposite microplate in the plane stress state are expressed in the following form (Mohammadimehr et al., 2016a,b, 2017; Ghorbanpour Arani et al., 2016)

$$
\begin{gathered}
\left\{\begin{array}{l}
\sigma_{11} \\
\sigma_{22} \\
\tau_{12} \\
\tau_{13} \\
\tau_{23}
\end{array}\right\}=\frac{1}{1-\left(e_{o} a\right)^{2} \nabla^{2}}\left(\left[\begin{array}{ccccc}
C_{11} & C_{12} & 0 & 0 & 0 \\
C_{12} & C_{22} & 0 & 0 & 0 \\
0 & 0 & C_{44} & 0 & 0 \\
0 & 0 & 0 & C_{44} & 0 \\
0 & 0 & 0 & 0 & C_{55}
\end{array}\right]\left\{\begin{array}{l}
\varepsilon_{11} \\
\varepsilon_{22} \\
\gamma_{12} \\
\gamma_{13} \\
\gamma_{23}
\end{array}\right\}\right. \\
\left.-\left[\begin{array}{ccc}
0 & 0 & e_{31} \\
0 & 0 & e_{31} \\
0 & e_{24} & 0 \\
e_{15} & 0 & 0 \\
0 & 0 & 0
\end{array}\right]\left\{\begin{array}{l}
E_{x} \\
E_{y} \\
E_{z}
\end{array}\right\}-\left[\begin{array}{ccc}
0 & 0 & f_{31} \\
0 & 0 & f_{31} \\
0 & f_{24} & 0 \\
f_{15} & 0 & 0 \\
0 & 0 & 0
\end{array}\right]\left\{\begin{array}{l}
H_{x} \\
H_{y} \\
H_{z}
\end{array}\right\}\right)
\end{gathered}
$$




$$
\begin{aligned}
& \left\{\begin{array}{l}
D_{x} \\
D_{y} \\
D_{z}
\end{array}\right\}=\frac{1}{1-\left(e_{o} a\right)^{2} \nabla^{2}}\left(\left[\begin{array}{ccccc}
0 & 0 & 0 & e_{15} & 0 \\
0 & 0 & e_{24} & 0 & 0 \\
e_{31} & e_{31} & 0 & 0 & 0
\end{array}\right]\left\{\begin{array}{l}
\varepsilon_{11} \\
\varepsilon_{22} \\
\gamma_{12} \\
\gamma_{13} \\
\gamma_{23}
\end{array}\right\}\right. \\
& \left.+\left[\begin{array}{ccc}
h_{11} & 0 & 0 \\
0 & h_{22} & 0 \\
0 & 0 & h_{33}
\end{array}\right]\left\{\begin{array}{l}
E_{x} \\
E_{y} \\
E_{z}
\end{array}\right\}+\left[\begin{array}{ccc}
g_{11} & 0 & 0 \\
0 & g_{22} & 0 \\
0 & 0 & g_{33}
\end{array}\right]\left\{\begin{array}{l}
H_{x} \\
H_{y} \\
H_{z}
\end{array}\right\}\right) \\
& \left\{\begin{array}{l}
B_{x} \\
B_{y} \\
B_{z}
\end{array}\right\}=\frac{1}{1-\left(e_{o} a\right)^{2} \nabla^{2}}\left(\left[\begin{array}{ccccc}
0 & 0 & 0 & f_{15} & 0 \\
0 & 0 & f_{24} & 0 & 0 \\
f_{31} & f_{31} & 0 & 0 & 0
\end{array}\right]\left\{\begin{array}{c}
\varepsilon_{11} \\
\varepsilon_{22} \\
\gamma_{12} \\
\gamma_{13} \\
\gamma_{23}
\end{array}\right\}\right. \\
& \left.+\left[\begin{array}{ccc}
g_{11} & 0 & 0 \\
0 & g_{22} & 0 \\
0 & 0 & g_{33}
\end{array}\right]\left\{\begin{array}{l}
E_{x} \\
E_{y} \\
E_{z}
\end{array}\right\}+\left[\begin{array}{ccc}
\mu_{11} & 0 & 0 \\
0 & \mu_{22} & 0 \\
0 & 0 & \mu_{33}
\end{array}\right]\left\{\begin{array}{l}
H_{x} \\
H_{y} \\
H_{z}
\end{array}\right\}\right)
\end{aligned}
$$

where $\sigma_{11}, \sigma_{22}$ and $\varepsilon_{11}, \varepsilon_{22}$ are the normal stresses and strains, respectively. $\tau_{12}, \tau_{13}, \tau_{23}$ and $\gamma_{12}, \gamma_{13}, \gamma_{23}$ denote the shear stresses and strains, respectively. $C_{i j}, e_{i j}, f_{i j}$ and $g_{i j}$ denote elastic, piezoelectric, piezomagnetic and magnetoelectric constants, respectively; $h_{i j}$ and $\mu_{i j}$ are dielectric and magnetic permeability coefficients, respectively. $E_{i j}$ and $H_{i j}$ are the electric magnetic field intensity, respectively.

The electric and magnetic fields are considered in terms of electric and magnetic potentials $\phi$ and $\varphi$, respectively, which are defined as follows

$$
E_{i}=-\phi_{, i} \quad H_{i}=-\varphi_{, i} \quad i=1,2,3
$$

\section{The governing equations of motion for the MEE nanocomposite microplate}

The governing differential equations of motion for the MEE nanocomposite microplate are derived using Hamilton's principle which is given by (Mohammadimehr and Mostafavifar, 2016)

$$
\int_{0}^{t}(\delta T-\delta U-\delta W) d t=0
$$

where $\delta T, \delta U$ and $\delta W$ are the variations of kinetic energy and strain energy, the work done by external applied forces, respectively.

Variations of the kinetic energy for a sandwich plate can be described as follows (Ghorbanpour and Haghparast, 2017)

$$
\delta T=\int_{V} \rho_{i} \frac{\partial u_{i}}{\partial t} \delta\left(\frac{\partial u_{i}}{\partial t}\right) d V=\int_{A} \int_{\frac{-h}{2}}^{\frac{h}{2}} \rho_{i}\left(\dot{u}_{1} \delta \dot{u}_{1}+\dot{u}_{2} \delta \dot{u}_{2}+\dot{u}_{3} \delta \dot{u}_{3}\right) d z d A
$$

where

$$
I_{i}=\int_{-h}^{h} \rho z^{i} d z \quad(i=1,2,3,4,6) \quad C_{1}=\frac{4}{3 h^{2}}
$$

Variations of the strain energy for the MEE nanocomposite microplate can be expressed as 


$$
\begin{aligned}
\delta U & =\int_{V}\left(\sigma_{i j} \delta \varepsilon_{i j}-D_{i} \delta E_{i}-B_{i} H_{i}\right) d V \\
& =\int_{V}\left[\left(\sigma_{11} \delta \varepsilon_{11}+\sigma_{22} \delta \varepsilon_{22}+\sigma_{33} \delta \varepsilon_{33}+\tau_{12} \delta \gamma_{12}+\tau_{13} \delta \gamma_{13}+\tau_{23} \delta \gamma_{23}\right)\right. \\
& \left.-\left(D_{x} \delta E_{x}+D_{y} \delta E_{y}+D_{y} \delta E_{y}\right)-\left(B_{x} \delta H_{x}+B_{y} \delta H_{y}+B_{y} \delta H_{y}\right)\right] d V
\end{aligned}
$$

Variations of the work can be considered as follows

$$
\delta W=-\int P(x, y) \delta w d x+\int\left(k_{w} w-k_{G} \nabla^{2} w\right) \delta w d x
$$

where $K_{w}$ and $K_{G}$ are the transverse and shear coefficients of elastic medium, respectively.

By substituting Eqs. (4.2)-(4.4) into Eq. (4.1), the equilibrium equations of the MEE nanocomposite microplate resting on an elastic foundation can be obtained in the following form

$$
\begin{array}{ll}
\delta u: \quad N_{1, x}+N_{6, y}=I_{0} \ddot{u}+I_{1} \ddot{\psi}_{x}-C_{1} I_{3}\left(\ddot{\psi}_{x}+\frac{\partial \ddot{w}}{\partial x}\right) \\
\delta v: \quad N_{2, y}+N_{6, x}=I_{0} \ddot{v}+I_{1} \ddot{\psi}_{y}-C_{1} I_{3}\left(\ddot{\psi}_{y}+\frac{\partial \ddot{w}}{\partial y}\right) \\
\delta \psi_{x}: \quad M_{1, x}+M_{6, y}-Q_{1}-\frac{4}{3 h^{2}}\left(P_{1, x}+P_{6, y}\right)+\frac{4 \lambda}{h^{2}} R_{1} \\
=I_{1} \ddot{u}+I_{2} \ddot{\psi}_{x}-C_{1}\left(I_{3} \ddot{u}+2 I_{4} \ddot{\psi}_{x}+I_{4} \frac{\partial \ddot{w}}{\partial x}\right)+C_{1}^{2} I_{6}\left(\ddot{\psi_{x}}+\frac{\partial \ddot{w}}{\partial x}\right) \\
\delta \psi_{y}: \quad M_{2, y}+M_{6, x}-Q_{2}-\frac{4}{3 h^{2}}\left(P_{2, y}+P_{6, x}\right)+\frac{4}{h^{2}} R_{2} \\
\quad=I_{1} \ddot{v}+I_{2} \ddot{\psi}_{y}-C_{1}\left(I_{3} \ddot{v}+2 I_{4} \ddot{\psi}_{y}+I_{4} \frac{\partial \ddot{w}}{\partial y}\right)+C_{1}^{2} I_{6}\left(\ddot{\psi}_{x}+\frac{\partial \ddot{w}}{\partial y}\right) \\
\delta w: \quad Q_{1, x}+Q_{2, y}+\frac{4}{3 h^{2}}\left(P_{1, x x}+P_{2, y y}+2 P_{6, x y}\right)-\frac{4}{h^{2}}\left(R_{2, y}+R_{1, x}\right) \\
+\left(-k_{w} w+k_{G} \nabla^{2} w\right)+P(x, y)=C_{1} I_{3}\left(\frac{\partial \ddot{u}}{\partial x}+\frac{\partial \ddot{v}}{\partial y}\right) \\
+C_{1} I_{4}\left(\frac{\partial \ddot{\psi}_{x}}{\partial x}+\frac{\partial \ddot{\psi_{y}}}{\partial y}\right)+C_{1}^{2} I_{6}\left(\frac{\partial \ddot{\psi}_{x}}{\partial x}+\frac{\partial \ddot{\psi_{y}}}{\partial y}-\frac{\partial^{2} \ddot{w}}{\partial x^{2}}-\frac{\partial^{2} \ddot{w}}{\partial y^{2}}\right)+I_{0} \ddot{w}
\end{array}
$$

and

$$
\frac{\partial D_{z}}{\partial z}=0 \quad \frac{\partial B_{z}}{\partial z}=0
$$

where $N_{i}, M_{i}(i=1,2,6)$ denote the resultant forces and moments, respectively. $R_{i}, P_{i}$ are higher order resultant shear forces and moments, respectively, and $Q_{i}$ are transverse shear forces which are all defined by the following expressions

$$
\begin{aligned}
& \left(\left\{\begin{array}{l}
N_{1} \\
N_{2} \\
N_{6}
\end{array}\right\},\left\{\begin{array}{l}
M_{1} \\
M_{2} \\
M_{6}
\end{array}\right\},\left\{\begin{array}{l}
P_{1} \\
P_{2} \\
P_{6}
\end{array}\right\}\right)=\int_{\frac{-h}{2}}^{\frac{h}{2}}\left\{\begin{array}{l}
\sigma_{11} \\
\sigma_{22} \\
\tau_{12}
\end{array}\right\}\left(1, z, z^{3}\right) d z \\
& \left(\left\{\begin{array}{l}
Q_{1} \\
Q_{2}
\end{array}\right\},\left\{\begin{array}{l}
R_{1} \\
R_{2}
\end{array}\right\}\right)=\int_{\frac{-h}{2}}^{\frac{h}{2}}\left\{\begin{array}{l}
\tau_{13} \\
\tau_{23}
\end{array}\right\}\left(1, z^{2}\right) d z
\end{aligned}
$$

By substituting Eqs. (3.3) into Eqs. (4.6), the electric and magnetic potential are obtained which electric and magnetic boundary conditions assumed as follows 


$$
\begin{aligned}
\phi\left(\frac{h}{2}\right) & =\phi\left(-\frac{h}{2}\right)=0 & \phi & =\lambda_{1} \Delta_{1}\left(\frac{z^{2}}{2}-\frac{z^{4}}{3 h^{2}}-\frac{23 h^{2}}{192}\right)+\lambda_{1} \Delta_{2}\left(\frac{h^{2}}{192}-\frac{z^{4}}{3 h^{2}}\right) \\
\varphi\left(\frac{h}{2}\right) & =\varphi\left(-\frac{h}{2}\right)=0 & \varphi & =\lambda_{2} \Delta_{1}\left(\frac{z^{2}}{2}-\frac{z^{4}}{3 h^{2}}-\frac{23 h^{2}}{192}\right)+\lambda_{2} \Delta_{2}\left(\frac{h^{2}}{192}-\frac{z^{4}}{3 h^{2}}\right)
\end{aligned}
$$

where

$$
\lambda_{1}=\frac{e_{31}-\frac{g_{33} f_{31}}{\mu_{33}}}{h_{33}-\frac{g_{33}^{2}}{\mu_{33}}} \quad \lambda_{2}=\frac{f_{31}-g_{33} \lambda_{1}}{\mu_{33}} \quad \Delta_{1}=\psi_{x, x}+\psi_{y, y} \quad \Delta_{2}=w_{, x x}+w_{, y y}
$$

By substituting Eqs. (4.7) into Eqs. (4.5) and (4.6), the governing equations of motion for the MEE nanocomposite microplate based on TSDT are obtained as follows

$$
\begin{aligned}
\delta u: & A_{11} u_{, x x}+\left(A_{12}+A_{66}\right) v_{, x y}+A_{66} u_{, y y}=I_{0} \ddot{u}-e_{0}^{2} a^{2} I_{0}\left(\ddot{u}_{, x x}+\ddot{u}_{, y y}\right)+\left(I_{1}-C_{1} I_{3}\right) \ddot{\psi}_{x} \\
& -\left(I_{1}-C_{1} I_{3}\right) e_{0}^{2} a^{2}\left(\ddot{\psi}_{x, x x}+\ddot{\psi}_{x, y y}\right)-C_{1} I_{3} \ddot{w}_{, x}+C_{1} I_{3} e_{0}^{2} a^{2}\left(\ddot{w}_{, x x x}+\ddot{w}_{, x y y}\right)
\end{aligned}
$$

$\delta v: \quad A_{22} v_{, y y}+\left(A_{12}+A_{66}\right) u_{, x y}+A_{66} v_{, x x}=I_{0} \ddot{v}-e_{0}^{2} a^{2} I_{0}\left(\ddot{v}_{, x x}+\ddot{v}_{, y y}\right)+\left(I_{1}-C_{1} I_{3}\right) \ddot{\psi}_{y}$

$-\left(I_{1}-C_{1} I_{3}\right) e_{0}^{2} a^{2}\left(\ddot{\psi}_{y, x x}+\ddot{\psi}_{y, y y}\right)-C_{1} I_{3} \ddot{w}_{, y}+C_{1} I_{3} e_{0}^{2} a^{2}\left(\ddot{w}_{, y x x}+\ddot{w}_{, y y y}\right)$

$\delta \psi_{x}:\left(B_{11}-\frac{4 H_{11}}{3 h^{2}}\right) \psi_{x, x x}+\left(F_{11}-\frac{4 L_{11}}{3 h^{2}}\right) \psi_{x, y y}+\left(\frac{4 T_{22}}{3 h^{2}}-T_{11}\right) \psi_{x}$

$$
+\left(F_{11}+B_{12}-\frac{4 H_{12}}{3 h^{2}}-\frac{4 L_{11}}{3 h^{2}}\right) \psi_{y, x y}+\left(\frac{4 K_{11}}{3 h^{2}}-D_{11}\right) w_{, x x x}
$$

$+\left(\frac{4 L_{12}}{3 h^{2}}+\frac{4 K_{12}}{3 h^{2}}-D_{12}-F_{12}\right) w_{, x y y}+\left(\frac{4 T_{22}}{3 h^{2}}-T_{11}\right) w_{, x}$

$$
=\left(I_{1}-C_{1} I_{3}\right) \ddot{u}-\left(I_{1}-C_{1} I_{3}\right) e_{0}^{2} a^{2}\left(\ddot{u}_{, x x}+\ddot{u}_{, y y}\right)+\left(I_{2}-2 C_{1} I_{4}+C_{1}^{2} I_{6}\right) \ddot{\psi}_{x}
$$$$
+\left(2 C_{1} I_{4}+C_{1}^{2} I_{6}-I_{2}\right) e_{0}^{2} a^{2}\left(\ddot{\psi}_{x, x x}+\ddot{\psi}_{x, y y}\right)+\left(C_{1}^{2} I_{6}-C_{1} I_{4}\right) \ddot{w}_{, x}
$$$$
+\left(C_{1} I_{4}-C_{1}^{2} I_{6}\right) e_{0}^{2} a^{2} \ddot{w}_{, x x x}+\left(C_{1} I_{4}-C_{1}^{2} I_{6}\right) e_{0}^{2} a^{2} \ddot{w}_{, x y y}
$$

$\delta \psi_{y}:\left(B_{22}-\frac{4 H_{22}}{3 h^{2}}\right) \psi_{y, y y}+\left(F_{11}-\frac{4 L_{11}}{3 h^{2}}\right) \psi_{y, x x}+\left(\frac{4 T_{22}}{3 h^{2}}-T_{11}\right) \psi_{y}$

$$
\begin{aligned}
& +\left(F_{11}+B_{12}-\frac{4 H_{12}}{3 h^{2}}-\frac{4 L_{11}}{3 h^{2}}\right) \psi_{x, x y}+\left(\frac{4 K_{22}}{3 h^{2}}-D_{11}\right) w_{, y y y} \\
& +\left(\frac{4 L_{12}}{3 h^{2}}+\frac{4 K_{12}}{3 h^{2}}-D_{12}-F_{12}\right) w_{, x x y}+\left(\frac{4 T_{22}}{3 h^{2}}-T_{11}\right) w_{, y} \\
& =\left(I_{1}-C_{1} I_{3}\right) \ddot{v}-\left(I_{1}-C_{1} I_{3}\right) e_{0}^{2} a^{2}\left(\ddot{v}_{, x x}+\ddot{v}_{, y y}\right)+\left(I_{2}-2 C_{1} I_{4}+C_{1}^{2} I_{6}\right) \ddot{\psi}_{y} \\
& +\left(2 C_{1} I_{4}+C_{1}^{2} I_{6}-I_{2}\right) e_{0}^{2} a^{2}\left(\ddot{\psi}_{y, x x}+\ddot{\psi}_{y, y y}\right)+\left(C_{1}^{2} I_{6}-C_{1} I_{4}\right) \ddot{w}_{, y} \\
& +\left(C_{1} I_{4}-C_{1}^{2} I_{6}\right) e_{0}^{2} a^{2} \ddot{w}_{, y x x}+\left(C_{1} I_{4}-C_{1}^{2} I_{6}\right) e_{0}^{2} a^{2} \ddot{w}_{, y y y}
\end{aligned}
$$

$\delta w:\left(T_{11}-\frac{4 T_{22}}{h^{2}}\right) \psi_{x, x}+\left(T_{11}-\frac{4 T_{22}}{h^{2}}\right) \psi_{y, y}+\frac{4 H_{11}}{3 h^{2}} \psi_{x, x x x}+\frac{4 H_{22}}{3 h^{2}} \psi_{y, y y y}$

$$
\begin{aligned}
& +\left(\frac{4 H_{12}}{3 h^{2}}+\frac{8 L_{11}}{3 h^{2}}\right)\left(\psi_{x, x y y}+\psi_{y, x x y}\right)+\left(T_{11}-\frac{4 T_{22}}{h^{2}}+e_{0}^{2} a^{2} K_{w}-K_{G}\right)\left(w_{, x x}+w_{, y y}\right) \\
& +\left(e_{0}^{2} a^{2} K_{G}-\frac{4 K_{11}}{3 h^{2}}\right) w_{, x x x x}+\left(e_{0}^{2} a^{2} K_{G}-\frac{4 K_{22}}{3 h^{2}}\right) w_{, y y y y}-\left(\frac{8 K_{12}}{3 h^{2}}+\frac{8 L_{12}}{3 h^{2}}\right) w_{, x x y y} \\
& -K_{w} w+P(x, y)=C_{1} I_{3} \ddot{u}_{, x}-C_{1} I_{3} e_{0}^{2} a^{2}\left(\ddot{u}_{, x x x}+\ddot{u}_{, x y y}\right)+C_{1} I_{3} \ddot{v}_{, x} \\
& -C_{1} I_{3} e_{0}^{2} a^{2}\left(\ddot{v}_{, x x x}+\ddot{v}_{, x y y}\right)+\left(C_{1} I_{4}+C_{1}^{2} I_{6}\right) \ddot{\psi}_{x, x}-\left(C_{1} I_{4}+C_{1}^{2} I_{6}\right) e_{0}^{2} a^{2}\left(\ddot{\psi}_{x, x x x}+\ddot{\psi}_{x, x y y}\right) \\
& +\left(C_{1} I_{4}+C_{1}^{2} I_{6}\right) \ddot{\psi}_{y, y}-\left(C_{1} I_{4}+C_{1}^{2} I_{6}\right) e_{0}^{2} a^{2}\left(\ddot{\psi}_{y, y x x}+\ddot{\psi}_{y, y y y}\right)-\left(C_{1}^{2} I_{6}+e_{0}^{2} a^{2}\right) \ddot{w}_{, x x} \\
& -\left(C_{1}^{2} I_{6}+e_{0}^{2} a^{2}\right) \ddot{w}_{, y y}+e_{0}^{2} a^{2} C_{1} I_{6}\left(\ddot{w}_{, x x x x}+\ddot{w}_{, y y y y}\right)+2 e_{0}^{2} a^{2} C_{1} I_{6} \ddot{w}_{, x x y y}+I_{0} \ddot{w}
\end{aligned}
$$


where the above coefficients are defined in Appendix A.

Substituting Eqs. (4.8) into Eq. (3.3), the electric and magnetic field is written as

$$
\begin{aligned}
& E_{z}=\lambda_{1}\left(z-\frac{4 z^{3}}{3 h^{2}}\right)\left(\psi_{x, x}+\psi_{x, x}\right)-\frac{4 z^{3}}{3 h^{2}} \lambda_{1}\left(w_{, x x}+w_{, y y}\right) \\
& H_{z}=\lambda_{2}\left(z-\frac{4 z^{3}}{3 h^{2}}\right)\left(\psi_{x, x}+\psi_{x, x}\right)-\frac{4 z^{3}}{3 h^{2}} \lambda_{2}\left(w_{, x x}+w_{, y y}\right)
\end{aligned}
$$

\section{Navier's type solution for the MEE nanocomposite microplate}

Analytical solutions for a simply supported rectangular MEE nanocomposite microplate are obtained using Navier's solution technique. Using Navier's solution, the displacements of the microplate can be written as follows (Mohammadimehr et al., 2016a)

$$
\begin{aligned}
& u(x, y, t)=\sum_{m=1}^{\infty} \sum_{n=1}^{\infty} U_{m n} \cos (\alpha x) \sin (\beta y) \mathrm{e}^{\mathrm{i} \omega t} \\
& v(x, y, t)=\sum_{m=1}^{\infty} \sum_{n=1}^{\infty} V_{m n} \sin (\alpha x) \cos (\beta y) \mathrm{e}^{\mathrm{i} \omega t} \\
& \psi_{x}(x, y, t)=\sum_{m=1}^{\infty} \sum_{n=1}^{\infty} \Psi_{x} m n \cos (\alpha x) \sin (\beta y) \mathrm{e}^{\mathrm{i} \omega t} \\
& \psi_{y}(x, y, t)=\sum_{m=1}^{\infty} \sum_{n=1}^{\infty} \Psi_{y} m n \sin (\alpha x) \cos (\beta y) \mathrm{e}^{\mathrm{i} \omega t} \\
& w(x, y, t)=\sum_{m=1}^{\infty} \sum_{n=1}^{\infty} W_{m n} \sin (\alpha x) \sin (\beta y) \mathrm{e}^{\mathrm{i} \omega t}
\end{aligned}
$$

where $\alpha$ and $\beta$ are equal to $m \pi / l, n \pi / b$, respectively.

\subsection{Free vibration analysis of the nanocomposite microplate}

The matrix form of free vibration equations of the microplate is written as

$$
\left(\mathbf{S}-\omega^{2} \mathbf{M}\right) \mathbf{U}=\mathbf{0}
$$

where the non-zero elements of the mass and stiffness matrix are given in Appendix B.

\subsection{Buckling analysis of the nanocomposite microplate} follows

The matrix form of buckling equations for the nanocomposite microplate can be written as

$$
\begin{aligned}
& {\left[\begin{array}{llllc}
S_{11} & S_{12} & S_{13} & S_{14} & S_{15} \\
S_{21} & S_{22} & S_{23} & S_{24} & S_{25} \\
S_{31} & S_{32} & S_{33} & S_{34} & S_{35} \\
S_{41} & S_{42} & S_{43} & S_{44} & S_{45} \\
S_{51} & S_{52} & S_{53} & S_{54} & S_{55}-N_{0}\left(\alpha^{2}+k \beta^{2}\right)
\end{array}\right]\left\{\begin{array}{c}
U_{m n} \\
V_{m n} \\
\Psi_{x} m n \\
\Psi_{y} m n \\
W_{m n}
\end{array}\right\}=\left\{\begin{array}{l}
0 \\
0 \\
0 \\
0 \\
0
\end{array}\right\}} \\
& \overline{\mathbf{C}}=\left[\begin{array}{llll}
S_{11} & S_{12} & S_{13} & S_{14} \\
S_{21} & S_{22} & S_{23} & S_{24} \\
S_{31} & S_{32} & S_{33} & S_{34} \\
S_{41} & S_{42} & S_{43} & S_{44}
\end{array}\right]^{-1} \quad k=\frac{N_{x x}}{N_{y y}}
\end{aligned}
$$


Using Eq. (5.3), we obtain an expression for the critical buckling load $N_{0}$ of the MEE nanocomposite microplate

$$
N_{0}=\frac{1}{\alpha^{2}+k \beta^{2}}\left(S_{55}-\left\{\begin{array}{llll}
S_{51} & S_{52} & S_{53} & S_{54}
\end{array}\right\} \overline{\mathbf{C}}\left\{\begin{array}{llll}
S_{15} & S_{25} & S_{35} & S_{45}
\end{array}\right\}^{\mathrm{T}}\right)
$$

\subsection{Forced vibration of the nanocomposite microplate}

The load $P(x, y, t)$ can be exoressed in the form of series

$$
p(x, y, t)=\sum_{m=1}^{\infty} \sum_{n=1}^{\infty} P_{0} \sin (\Omega t) \sin (\alpha x) \sin (\beta y)
$$

where $\Omega$ is the frequency of forced vibration. The equation of motion for the MEE nanocomposite microplate will then include a variable, time-dependent, transverse load $p(x, y, t)$.

The matrix form of the response system equations for the MEE microplate is obtained as follows

$$
\left\{\begin{array}{lllll}
U_{m} & V_{m} & \Psi_{x} m n & \Psi_{y} m n & W_{m n}
\end{array}\right\}^{\mathrm{T}}=\frac{1}{\omega_{n}^{2}-\Omega^{2}} \mathbf{M}^{-1}\left[\begin{array}{ccccc}
0 & 0 & 0 & 0 & P_{0}
\end{array}\right]^{\mathrm{T}}
$$

\subsection{Dimensionless parameter of the nanocomposite microplate}

The dimensionless deflection, natural frequency and buckling load of the MEE nanocomposite microplate is written as follows

$$
\bar{W}=\frac{C_{i j \max } h^{3} w}{P_{0} l^{4}} \quad \bar{\omega}=\sqrt{\frac{\rho l^{4} \omega}{C_{i j \max } h^{2}}} \quad \bar{N}=\frac{l^{2} N_{0}}{C_{i j \max } h^{3}}
$$

\section{Numerical results and discussions}

The piezoelectric and piezomagentic properties of the $\mathrm{BaTiO}_{3}$ (inclusion)- $\mathrm{CoFe}_{2} \mathrm{O}_{4}$ (matrix) nanocomposite microplate with different volume fractions $V_{f}$ of the inclusions can be found in Sih and Sog (2002), Song and Sih (2002). They are listed in Table 1.

Numerical results for bending, buckling, free and forced vibration are presented for the MEE nanocomposite microplate resting on a two-parameter elastic foundations with all edges simply supported.

To validate the results of this research with the literature, a single-layered MEE square thick plate, with $l=b=1 \mathrm{~m}, h=0.3 \mathrm{~m}$, simply-supported boundary conditions, and material properties given by Table 2 is considered. The dimensionless fundamental frequency is calculated

as $\bar{\omega}=\sqrt{\rho_{\max } / C_{i j \max }} l \omega$, where $C_{i j \max }$ and $\rho_{\max }$ are the maximum values of the stiffness coefficient and density of the layers, respectively. The results are shown in Table 2 along with some other published results.

Table 3 indicates the dimensionless biaxial buckling load of simply-supported square nanoplates. From this Table, it is observed that the presented results are in good agreement with those reported in the literature.

Table 4 presents the dimensionless center deflections of isotropic square plates under uniform loading. They are calculated with various side-to-thickness ratios up to $a / h=10000$, and compared to earlier studies.

The natural frequencies of the simply supported MEE nanocomposite microplate are obtained using Eq. (5.2). From Fig. 2a, it is seen that the volume fraction plays an important role for the MEE nanocomposite microplate in terms of the natural frequency, and its effects can 
Table 1. Properties of the $\mathrm{BaTiO}_{3}, \mathrm{CoFe}_{2} \mathrm{O}_{4}$ and $\mathrm{BaTiO}_{3}-\mathrm{CoFe}_{2} \mathrm{O}_{4}$ nanocomposite microplate with different volume fractions

\begin{tabular}{|c|c|c|c|c|c|c|c|}
\hline \multirow[t]{2}{*}{ Properties } & \multirow{2}{*}{$\begin{array}{c}\text { Piezoelectric } \\
\left(\mathrm{BaTiO}_{3}\right)\end{array}$} & \multirow{2}{*}{$\begin{array}{l}\text { Piezomagnetic } \\
\qquad\left(\mathrm{CoFe}_{2} \mathrm{O}_{4}\right)\end{array}$} & \multicolumn{5}{|c|}{$\begin{array}{l}V_{f}\left(\text { volume fraction for } \mathrm{CoFe}_{2} \mathrm{O}_{4} \text { in }\right. \\
\mathrm{BaTiO}_{3}-\mathrm{CoFe}_{2} \mathrm{O}_{4} \text { nanocomposite) }\end{array}$} \\
\hline & & & 0.1 & 0.3 & 0.5 & 0.7 & 0.9 \\
\hline$C_{11}[\mathrm{Gpa}]$ & 166 & 286 & 178.0 & 202 & 226 & 250.0 & 274 \\
\hline$C_{12}[\mathrm{Gpa}]$ & 77 & 173 & 87.2 & 105.7 & 124 & 142.7 & 161 \\
\hline$C_{22}[\mathrm{Gpa}]$ & 166 & 286 & 172.8 & 194.2 & 216 & 237.3 & 259 \\
\hline$C_{44}[\mathrm{Gpa}]$ & 43 & 45.3 & 43.2 & 43.7 & 44 & 44.6 & 45 \\
\hline$e_{31}\left[\mathrm{c} / \mathrm{m}^{2}\right]$ & 43 & 45.3 & -3.96 & -3.08 & -2.2 & -1.32 & -4.4 \\
\hline$e_{33}\left[\mathrm{c} / \mathrm{m}^{2}\right]$ & 44.5 & 56.5 & 16.74 & 13.02 & 9.3 & 5.58 & 1.86 \\
\hline$e_{15}\left[\mathrm{c} / \mathrm{m}^{2}\right]$ & -4.4 & 0 & 10.44 & 8.12 & 5.8 & 3.48 & 1.16 \\
\hline$h_{11}\left[\times 10^{-10} \mathrm{C}^{2} /\left(\mathrm{Nm}^{2}\right)\right]$ & 0 & 580.3 & 100.9 & 78.6 & 56.4 & 34.2 & 11.9 \\
\hline$h_{33}\left[\times 10^{-10} \mathrm{C}^{2} /\left(\mathrm{Nm}^{2}\right)\right]$ & -4.4 & 0 & 113.5 & 88.5 & 63.5 & 38.5 & 13.4 \\
\hline$f_{31}[\mathrm{~N} /(\mathrm{Am})]$ & 0 & 580.3 & 58.03 & 174.1 & 290.2 & 406.2 & 522.3 \\
\hline$f_{33}[\mathrm{~N} /(\mathrm{Am})]$ & 11.6 & 0 & 69.97 & 209.9 & 350.0 & 489.8 & 629.7 \\
\hline$f_{15}[\mathrm{~N} /(\mathrm{Am})]$ & 0 & 550 & 55.00 & 165.0 & 275.0 & 385.0 & 495.0 \\
\hline$\mu_{11}\left[\times 10^{-6} \mathrm{NS}^{2} / \mathrm{C}^{2}\right]$ & 11.6 & 0 & 63.5 & 180.5 & 297.0 & 414.5 & 531.5 \\
\hline$\mu_{33}\left[\times 10^{-6} \mathrm{NS}^{2} / \mathrm{C}^{2}\right]$ & 0 & 550 & 24.7 & 541.0 & 83.5 & 112.9 & 142.3 \\
\hline$\rho\left[\mathrm{kg} / \mathrm{m}^{3}\right]$ & 126 & 0.93 & 5750 & 5650 & 5550 & 5450 & 5350 \\
\hline
\end{tabular}

Table 2. Dimensionless fundamental frequencies of MEE plates

\begin{tabular}{|l|c|c|}
\hline \multirow{2}{*}{ Method } & \multicolumn{2}{|c|}{ Material } \\
\cline { 2 - 3 } & $\begin{array}{c}\text { Piezoelectric } \\
\mathrm{BaTiO}_{3}\end{array}$ & $\begin{array}{c}\text { Piezomagnetic } \\
\mathrm{CoFe}_{2} \mathrm{O}_{4}\end{array}$ \\
\hline \hline Wu and Lu (2009) & 1.2523 & 1.0212 \\
\hline Shooshtari and Razavi (2015) & 1.2426 & 1.1023 \\
\hline Present study & 1.2952 & 1.1130 \\
\hline
\end{tabular}

Table 3. Comparison of dimensionless biaxial buckling load $\left(N_{c r}=N_{0} a / D, D=E h^{3} /[12(1-\right.$ $\left.v^{2}\right)$ ] for square nanoplates with all edges simply-supported ( $\left.a=10 \mathrm{~nm}, a / h=2\right)$

\begin{tabular}{|l|c|c|}
\hline \multirow{2}{*}{\multicolumn{1}{|c|}{ Method }} & \multicolumn{2}{c|}{$e_{0} a[\mathrm{~nm}]$} \\
\cline { 2 - 3 } & 0 & 1 \\
\hline \hline Malekzadeh and Shojaee (2013) & 8.5249 & 7.1039 \\
\hline Wang and Wang (2011) & 8.4543 & 7.1533 \\
\hline Karimi et al. (2015) & 8.6052 & 7.2204 \\
\hline Present study & 8.5232 & 7.1138 \\
\hline
\end{tabular}

not be ignored for microplate. It is shown that by increasing the volume fraction, the dimensionless natural frequency increases. The reason is that a greater volume fraction makes the microplate stiffer. Figure $2 \mathrm{~b}$ depicts the effects of the Pasternak shear constant on the natural frequency. From this figure, it can be found that by increasing this parameter, the stiffness of the nanocomposite microplate increases and this result is similar to the dimensionless natural frequency. The effect of volume fraction on the deflection is shown in Fig. 3a. It is shown that an increase in the volume fraction will decrease the dimensionless deflection. The critical buckling loads of the MEE nanocomposite microplate are obtained using Eq. (5.4). Figure 3b depicts the variation of critical buckling load versus volume fraction. From this figure, it can be seen that with an increase in the volume fraction, the critical buckling load for all the length to width 
Table 4. Comparison of dimensionless center deflection $W\left(\frac{a}{2}, \frac{b}{2}\right) D /\left[\left(P_{0} a^{4}\right), D=E h^{3} /[12(1-\right.$ $\left.v^{2}\right)$ ] for simply-supported square isotropic plates under uniform loads

\begin{tabular}{|l|c|c|c|c|}
\hline \multirow{2}{*}{ Method } & \multicolumn{4}{|c|}{$a / h$} \\
\cline { 2 - 5 } & 10 & 100 & 1000 & 10000 \\
\hline \hline Nguyen et al. (2016) & 0.4272 & 0.4064 & 0.4062 & 0.4062 \\
\hline Nguyen-Xuan et al. (2008), MITC4 & 0.4273 & 0.4064 & 0.4062 & 0.4062 \\
\hline Nguyen-Xuan et al. (2008), MISC1 & 0.4273 & 0.4065 & 0.4063 & 0.4063 \\
\hline Taylor and Auricchio (1993) & 0.4273 & 0.4064 & 0.4062 & 0.4062 \\
\hline Present study & 0.4266 & 0.4055 & 0.4053 & 0.4053 \\
\hline
\end{tabular}

(a)

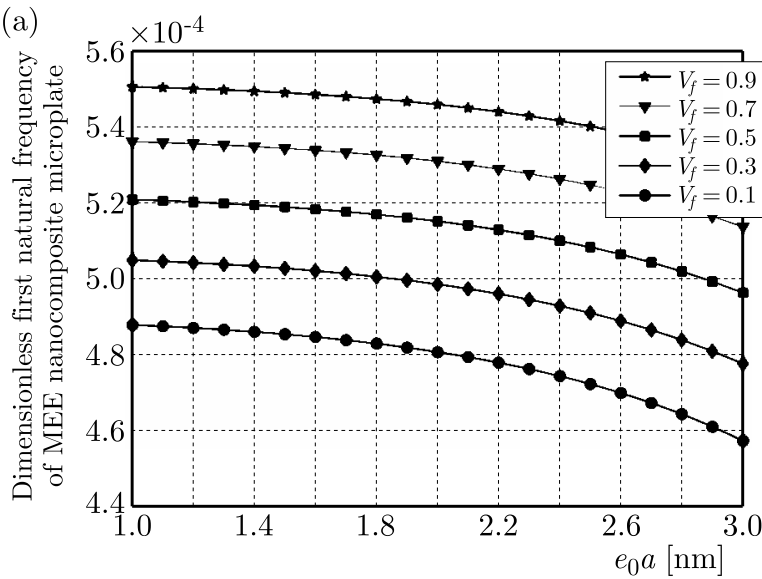

(b)

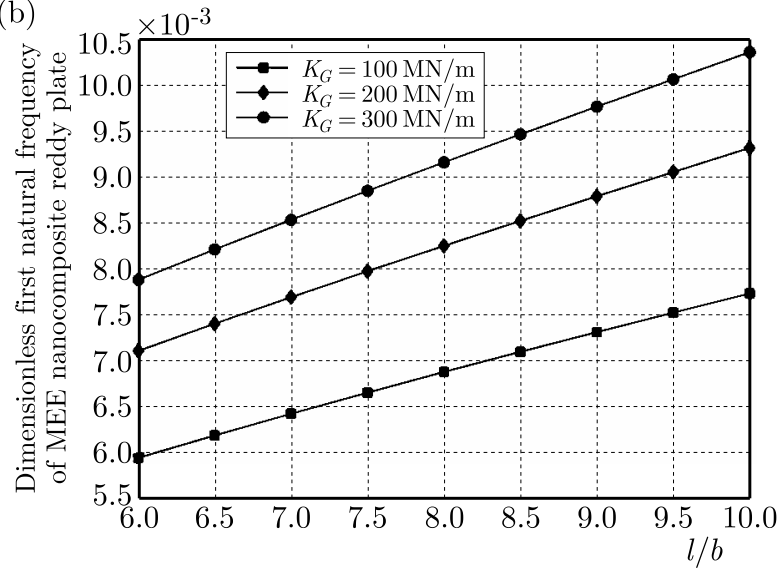

Fig. 2. (a) The effect of volume fraction on the dimensionless natural frequency: $l=4 \mu \mathrm{m}, b=4 \mu \mathrm{m}$, $h=0.04 \mu \mathrm{m}, K_{w}=0, K_{G}=0$. (b) The effect of the Pasternak shear constant on the dimensionless natural frequency: $l=400 \mu \mathrm{m}, h=80 \mu \mathrm{m}, V_{f}=0.5, K_{w}=0, e_{0} a=1 \mathrm{~nm}$

(a)

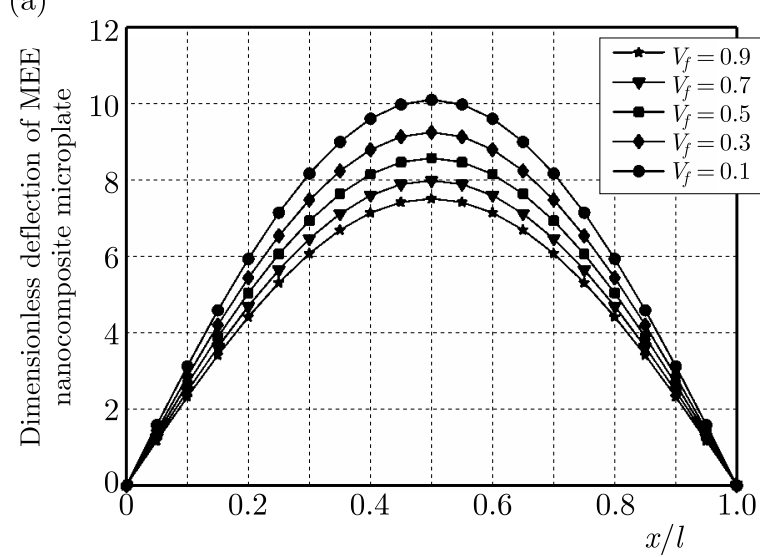

(b)

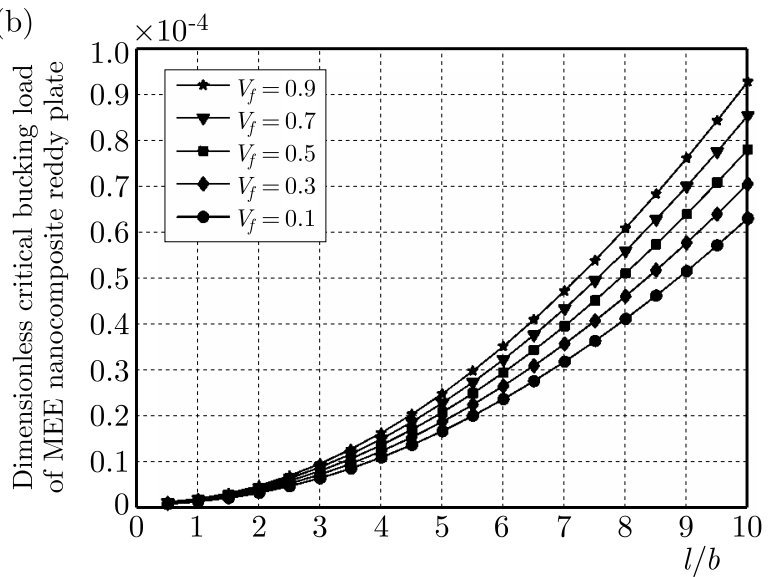

Fig. 3. (a) The effect of volume fraction on the dimensionless deflection: $l=400 \mu \mathrm{m}, b=400 \mu \mathrm{m}$, $h=80 \mu \mathrm{m}, K_{w}=0, K_{G}=0, P=100 \mathrm{~N} / \mathrm{m}^{2}, e_{0} a=2 \mathrm{~nm}$. (b) The effect of volume fraction on the dimensionless critical buckling load: $l=400 \mu \mathrm{m}, h=80 \mu \mathrm{m}, K_{w}=0, K_{G}=0, e_{0} a=1 \mathrm{~nm}$

ratios $l / b$ will increase. The influence of the length to thickness ratio $l / h$ is shown in Fig. 4a. This figure shows that by increasing the length to thickness ratio $l / h$, the dimensionless critical buckling load decreases. The response system of the MEE nanocomposite microplate is obtained using Eq. (5.6). Figure 4b indicates the response system of the MEE nanocomposite microplate and different values of the volume fraction. It is seen from the results that by increasing the excitation frequency to the natural frequency ratio $\Omega / \omega$, the amplitude of the nanocomposite 
microplate reinforced by $\mathrm{CoFe}_{2} \mathrm{O}_{4}-\mathrm{BaTiO}_{3}$ increases. Also, by increasing the volume fraction, the deflection to thickness ratio $w / h$ decreases. Figure 5 depicts the effects of volume fraction on the maximum deflection to thickness ratio $w_{\max } / h$. From this figure, it can be found that by increasing the volume fraction, the maximum deflection to thickness ratio $w_{\max } / h$ decreases.

(a)

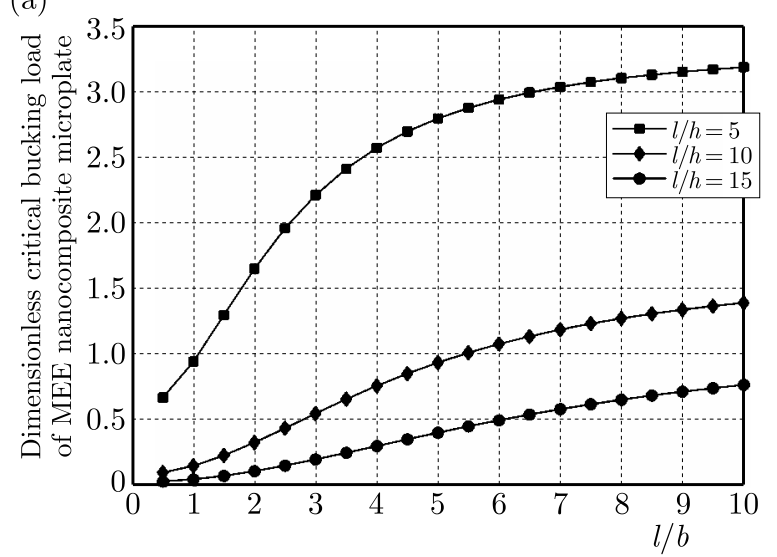

(b)

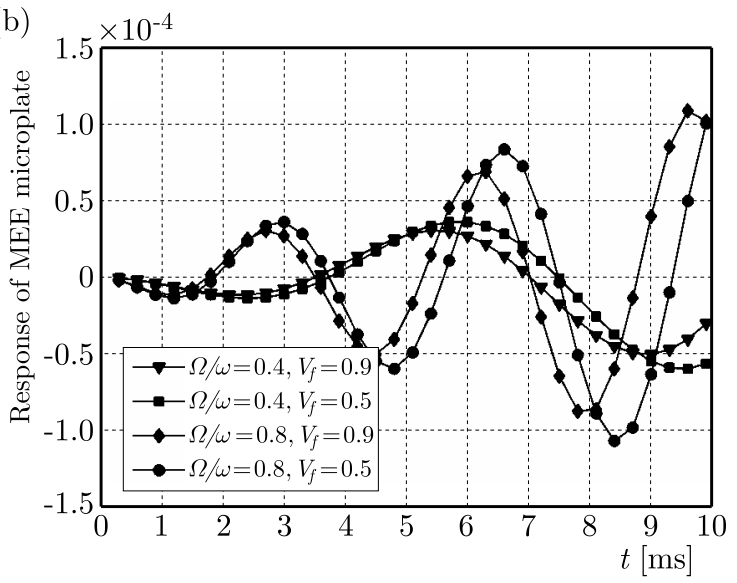

Fig. 4. (a) The effect of the length to thickness ratio $l / h$ on the critical buckling load: $l=400 \mu \mathrm{m}$, $V_{f}=0.5, K_{w}=0, K_{G}=0, e_{0} a=1 \mathrm{~nm}$. (b) The effect of volume fraction on the response system: $l=400 \mu \mathrm{m}, b=400 \mu \mathrm{m}, h=20 \mu \mathrm{m}, K_{w}=0, K_{G}=0, P_{0}=1 \mathrm{~N} / \mathrm{m}^{2}, e_{0} a=1 \mathrm{~nm}$

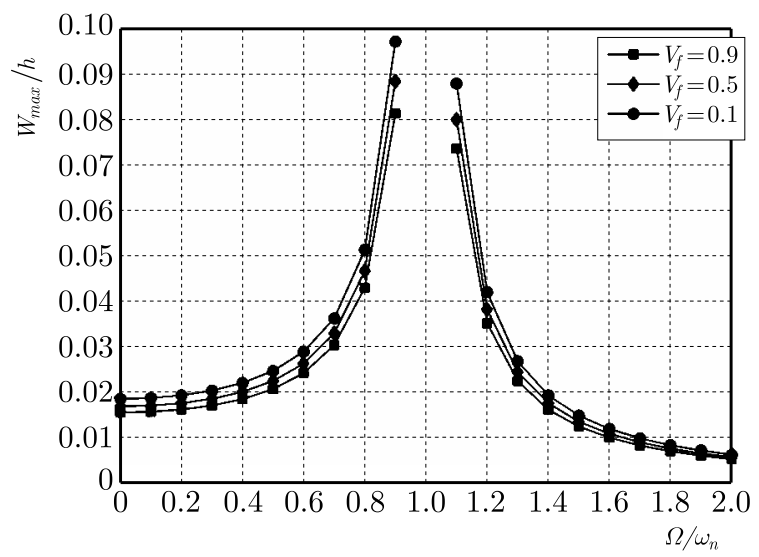

Fig. 5. The effect of volume fraction on the maximum deflection to thickness ratio $W_{\max } / h: l=4 \mu \mathrm{m}$, $b=4 \mu \mathrm{m}, h=0.1 \mu \mathrm{m}, K_{w}=0, K_{G}=0, P_{0}=1 \mathrm{~N} / \mathrm{m}^{2}, e_{0} a=1 \mathrm{~nm}$

Figure 6a shows that by increasing the spring constant of the Winkler type, the intensity of electric field decreases. Figure $6 \mathrm{~b}$ presents the influence of the Pasternak shear constant on the magnetic field, respectively. The results show that by increasing the elastic constant, the intensity of magnetic and electric field decreases.

\section{Conclusions}

A theoretical analysis on bending, buckling, free and forced vibration characteristics of an MEE nanocomposite microplate are carried out in the present work. The Hamilton principle, higher order shear deformation theory and Maxwell's equations are considered to derive the equations of motion and distribution of electrical potential, magnetic field along the thickness direction of the MEE nanocomposite microplate. Some conclusions of this research can be listed as follows:

- For the MEE nanocomposite microplate, the natural frequency and critical buckling load increases with the increasing volume fraction of $\mathrm{CoFe}_{2} \mathrm{O}_{4} \mathrm{O}_{4}-\mathrm{BaTiO}_{3}$, because the nanocomposite microplate becomes stiffer in such a case. 
(a)

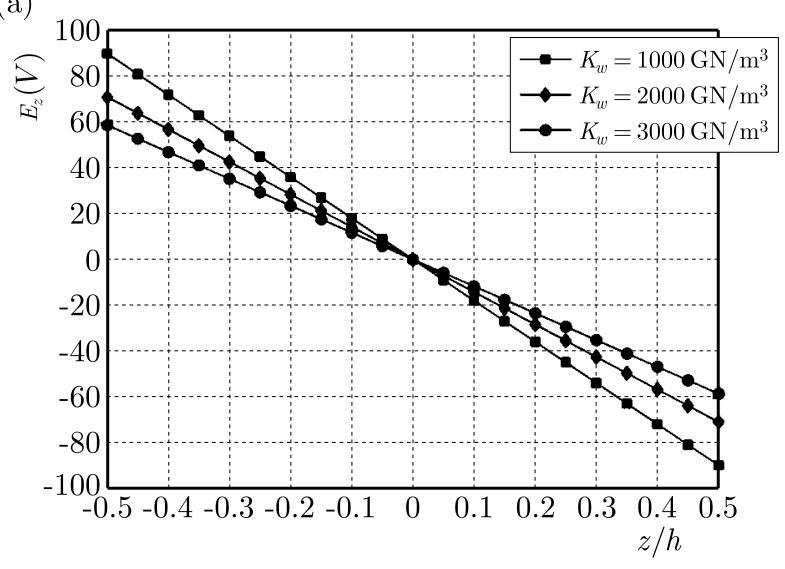

(b)

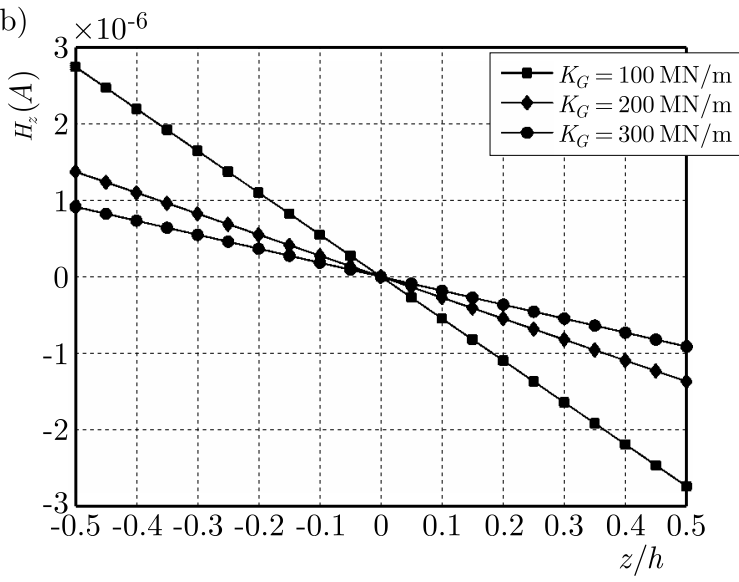

Fig. 6. (a) The effect of the Winkler spring constant on the intensity of electric field: $l=40 \mu \mathrm{m}$, $b=40 \mu \mathrm{m}, h=1 \mu \mathrm{m}, K_{G}=0, P=1 \mathrm{~N} / \mathrm{m}^{2}, e_{0} a=1 \mathrm{~nm}$. (b) The effect of the Pasternak shear constant on the intensity of magnetic field : $l=40 \mu \mathrm{m}, b=40 \mu \mathrm{m}, h=1 \mu \mathrm{m}, K_{w}=0, P=1 \mathrm{~N} / \mathrm{m}^{2}, e_{0} a=1 \mathrm{~nm}$

- The natural frequency and critical buckling load decreases, and also the maximum deflection, whereas the intensity of magnetic and electric fields increases with the decreasing Winkler and Pasternak shear constants of the MEE nanocomposite microplate.

- For the MEE nanocomposite microplate, the amplitude of vibration decreases with the increasing volume fraction.

\section{Appendix A}

$$
\begin{aligned}
& A_{11}=C_{11} h \quad A_{12}=C_{12} h \\
& A_{22}=C_{22} h \quad A_{66}=C_{66} h \\
& B_{11}=\frac{h^{3}}{15}\left(C_{11}+e_{31} \lambda_{1}+f_{31} \lambda_{2}\right) \\
& B_{12}=\frac{h^{3}}{15}\left(C_{12}+e_{31} \lambda_{1}+f_{31} \lambda_{2}\right) \\
& B_{22}=\frac{h^{3}}{15}\left(C_{22}+e_{31} \lambda_{1}+f_{31} \lambda_{2}\right) \\
& D_{11}=\frac{h^{3}}{60}\left(C_{11}+e_{31} \lambda_{1}+f_{31} \lambda_{2}\right) \\
& D_{12}=\frac{h^{3}}{60}\left(C_{12}+e_{31} \lambda_{1}+f_{31} \lambda_{2}\right) \\
& D_{22}=\frac{h^{3}}{60}\left(C_{22}+e_{31} \lambda_{1}+f_{31} \lambda_{2}\right) \\
& F_{11}=C_{66} \frac{h^{3}}{15} \quad F_{12}=C_{66} \frac{h^{3}}{30} \\
& H_{11}=\left(\frac{h^{5}}{80}-\frac{h^{5}}{336}\right)\left(C_{11}+e_{31} \lambda_{1}+f_{31} \lambda_{2}\right) \\
& H_{12}=\left(\frac{h^{5}}{80}-\frac{h^{5}}{336}\right)\left(C_{12}+e_{31} \lambda_{1}+f_{31} \lambda_{2}\right) \\
& H_{22}=\left(\frac{h^{5}}{80}-\frac{h^{5}}{336}\right)\left(C_{22}+e_{31} \lambda_{1}+f_{31} \lambda_{2}\right) \\
& K_{11}=\frac{h^{5}}{336}\left(C_{11}+e_{31} \lambda_{1}+f_{31} \lambda_{2}\right) \\
& K_{12}=\frac{h^{5}}{336}\left(C_{12}+e_{31} \lambda_{1}+f_{31} \lambda_{2}\right) \\
& K_{22}=\frac{h^{5}}{336}\left(C_{22}+e_{31} \lambda_{1}+f_{31} \lambda_{2}\right) \\
& L_{11}=\left(\frac{h^{5}}{80}-\frac{h^{5}}{336}\right) C_{66} \\
& L_{12}=\frac{h^{5}}{168} C_{66} \\
& T_{11}=\frac{2 h}{3} C_{44} \\
& T_{22}=\left(\frac{h^{3}}{12}-\frac{h^{3}}{20}\right) C_{44}
\end{aligned}
$$

\section{Appendix B}

$$
\begin{array}{lrl}
S_{11}=-A_{11} \alpha^{2}-A_{66} \beta^{2} & S_{12}=-\left(A_{12}+A_{66}\right) \alpha \beta \\
S_{21}=-\left(A_{12}+A_{66}\right) \alpha \beta & S_{22}=-A_{22} \beta^{2}-A_{66} \alpha^{2} \\
S_{33}=\left(B_{11}-\frac{4 H_{11}}{3 h^{2}}\right)\left(-\alpha^{2}\right)-\left(F_{11}-\frac{4 L_{11}}{3 h^{2}}\right) \beta^{2}+\frac{4 T_{22}}{3 h^{2}}-T_{11}
\end{array}
$$




$$
\begin{aligned}
& S_{34}=\left(B_{12}+F_{11}-\frac{4 H_{12}}{3 h^{2}}-\frac{4 L_{11}}{3 h^{2}}\right)(-\alpha \beta) \\
& S_{35}=\left(\frac{4 K_{11}}{3 h^{2}}-D_{11}\right)\left(-\alpha^{3}\right)-\left(\frac{4 L_{12}}{3 h^{2}}+\frac{4 K_{12}}{3 h^{2}}-D_{12}-F_{12}\right) \alpha \beta^{2}+\left(\frac{4 T_{22}}{3 h^{2}}-T_{11}\right) \alpha \\
& S_{43}=-\left(B_{12}+F_{11}-\frac{4 H_{12}}{3 h^{2}}-\frac{4 L_{11}}{3 h^{2}}\right) \alpha \beta \\
& S_{44}=-\left(B_{22}-\frac{4 H_{22}}{3 h^{2}}\right) \beta^{2}-\left(F_{11}-\frac{4 L_{11}}{3 h^{2}}\right) \alpha^{2}+\frac{4 T_{22}}{3 h^{2}}-T_{11} \\
& S_{45}=-\left(\frac{4 K_{22}}{3 h^{2}}-D_{22}\right) \beta^{3}-\left(\frac{4 L_{12}}{3 h^{2}}+\frac{4 K_{12}}{3 h^{2}}-D_{12}-F_{12}\right) \alpha^{2} \beta+\left(\frac{4 T_{22}}{3 h^{2}}-T_{11}\right) \beta \\
& S_{53}=\left(T_{11}-\frac{4 T_{22}}{h^{2}}\right)(-\alpha)-\frac{4 H_{11}}{3 h^{2}} \alpha^{3}+\left(\frac{4 H_{12}}{3 h^{2}}+\frac{8 L_{11}}{3 h^{2}}\right) \alpha \beta^{2} \\
& S_{54}=\left(T_{11}-\frac{4 T_{22}}{h^{2}}\right)(-\beta)-\frac{4 H_{22}}{3 h^{2}} \beta^{3}+\left(\frac{4 H_{12}}{3 h^{2}}+\frac{8 L_{11}}{3 h^{2}}\right) \alpha^{2} \beta \\
& S_{55}=-\left(T_{11}-\frac{4 T_{22}}{h^{2}}+e_{0}^{2} a^{2} K_{w}-K_{G}\right)\left(\alpha^{2}+\beta^{2}\right)+\left(e_{0}^{2} a^{2} K_{G}-\frac{4 K_{11}}{3 h^{2}}\right) \alpha^{4} \\
& +\left(e_{0}^{2} a^{2} K_{G}-\frac{4 K_{22}}{3 h^{2}}\right) \beta^{4}-\left(\frac{8 K_{12}}{3 h^{2}}+\frac{8 L_{12}}{3 h^{2}}\right) \alpha^{2} \beta^{2}-K_{w} \\
& m_{11}=-I_{0}-e_{0}^{2} a^{2} I_{0}\left(\alpha^{2}+\beta^{2}\right) \quad m_{13}=\left(-I_{1}-C_{1} I_{3}\right)-e_{0}^{2} a^{2}\left(I_{1}-C_{1} I_{3}\right)\left(\alpha^{2}+\beta^{2}\right) \\
& m_{15}=C_{1} I_{3} \alpha+C_{1} I_{3} e_{0}^{2} a^{2}\left(\alpha^{3}+\alpha \beta^{2}\right) \quad m_{22}=-I_{0}-I_{0} e_{0}^{2} a^{2}\left(\alpha^{2}+\beta^{2}\right) \\
& m_{24}=-\left(I_{1}-C_{1} I_{3}\right)-e_{0}^{2} a^{2}\left(I_{1}-C_{1} I_{3}\right)\left(\alpha^{2}+\beta^{2}\right) \quad m_{25}=C_{1} I_{3} \beta+e_{0}^{2} a^{2} C_{1} I_{3}\left(\alpha^{2} \beta+\beta^{3}\right) \\
& m_{31}=-\left(I_{1}-C_{1} I_{3}\right)+\left(C_{1} I_{3}-I_{1}\right) e_{0}^{2} a^{2}\left(\alpha^{2}+\beta^{2}\right) \\
& m_{33}=-\left(I_{2}-2 C_{1} I_{4}+C_{1}^{2} I_{6}\right)+\left(2 C_{1} I_{4}-C_{1}^{2} I_{6}-I_{2}\right) e_{0}^{2} a^{2}\left(\alpha^{2}+\beta^{2}\right) \\
& m_{35}=-\left(C_{1}^{2} I_{6}-C_{1} I_{4}\right) \alpha+\left(C_{1} I_{4}-C_{1}^{2} I_{6}\right) e_{0}^{2} a^{2}\left(\alpha^{3}+\alpha \beta^{2}\right) \\
& m_{42}=-\left(I_{1}-C_{1} I_{3}\right)+\left(C_{1} I_{3}-I_{1}\right) e_{0}^{2} a^{2}\left(\alpha^{2}+\beta^{2}\right) \\
& m_{44}=-\left(I_{2}-2 C_{1} I_{4}+C_{1}^{2} I_{6}\right)+\left(2 C_{1} I_{4}-C_{1}^{2} I_{6}-I_{2}\right) e_{0}^{2} a^{2}\left(\beta^{2}+\alpha^{2}\right) \\
& m_{45}=-\left(C_{1}^{2} I_{6}-C_{1} I_{4}\right) \beta+\left(C_{1} I_{4}-C_{1}^{2} I_{6}\right) e_{0}^{2} a^{2}\left(\beta^{3}+\alpha^{2} \beta\right) \\
& m_{51}=C_{1} I_{3} \alpha+C_{1} I_{3} e_{0}^{2} a^{2}\left(\alpha^{3}+\alpha \beta^{2}\right) \quad m_{52}=C_{1} I_{3} \beta+C_{1} I_{3} e_{0}^{2} a^{2}\left(\beta^{3}+\alpha^{2} \beta\right) \\
& m_{53}=\left(C_{1} I_{4}+C_{1}^{2} I_{6}\right) \alpha+\left(C_{1} I_{4}+C_{1}^{2} I_{6}\right) e_{0}^{2} a^{2} \alpha^{3}+\left(C_{1} I_{4}+C_{1}^{2} I_{6}\right) e_{0}^{2} a^{2} \alpha \beta^{2} \\
& m_{54}=\left(C_{1} I_{4}+C_{1}^{2} I_{6}\right) \beta+\left(C_{1} I_{4}+C_{1}^{2} I_{6}\right) e_{0}^{2} a^{2} \beta^{3}+\left(C_{1} I_{4}+C_{1}^{2} I_{6}\right) e_{0}^{2} a^{2} \alpha^{2} \beta \\
& m_{55}=-\left(C_{1}^{2} I_{6}+e_{0}^{2} a^{2}\right)\left(\alpha^{2}+\beta^{2}\right)-C_{1}^{2} I_{6} e_{0}^{2} a^{2}\left(\alpha^{4}+\beta^{4}\right)-2 C_{1}^{2} I_{6} e_{0}^{2} a^{2} \alpha^{2} \beta^{2}-I_{0}
\end{aligned}
$$

\section{Acknowledgments}

The authors would like to thank the referees for their valuable comments. They are also grateful to the Iranian Nanotechnology Development Committee for their financial support and University of Kashan for supporting this work by Grant No. 463855/4.

\section{References}

1. Arefi M., 2015, The effect of different functionalities of FGM and FGPM layers on free vibration analysis of the FG circular plates integrated with piezoelectric layers, Smart Structures and Systems, 15, 1345-1362

2. Chen J.Y., Heyliger P.R., PAn E., 2014, Free vibration of three-dimensional multilayered magneto-electro-elastic plates under combined clamped/free boundary conditions, Journal of Sound and Vibration, 333, 4017-4029

3. Du C., Li Y., JiN X., 2014, Nonlinear forced vibration of functionally graded cylindrical thin shells, Thin-Walled Structures, 78, 26-36 
4. Ebrahimi F., Nasirzadeh P., 2016, A nonlocal Timoshenko beam theory for vibration analysis of thick nonobeam using differential transform method, Journal of Theoretical and Applied Mechanics, 53, 1041-1053

5. Eringen A.C., 1983, On differential equations of nonlocal elasticity and solutions of screw dislocation and surface waves, Journal of Applied Physics, 54, 4703-4710

6. Eringen A.C., 2002, Nonlocal Continuum Field Theories, Springer-Verlag, New York

7. Ghorbanpour Arani A., Haghparast E., 2017, Vibration analysis of axially moving carbon nanotubereinforced composite plate under initial tension, Polymer Composites, 38, 4, 814-822

8. Ghorbanpour Arani A., Haghparast E., Baba Akbar Zarei H., 2016, Nonlocal vibration of axially moving graphene sheet resting on orthotropic visco-Pasternak foundation under longitudinal magnetic field, Physica B: Condensed Matter, 495, 35-49

9. Ghorbanpour Arani A., Rahnama Mobarakeh M., Shams Sh., Mohammadimehr M., 2012, The effect of CNT volume fraction on the magneto-thermo-electro-mechanical behavior of smart nanocomposite cylinder, Journal of Mechanical Science and Technology, 26, 2565-2572

10. Hasani Baferani A., Saidi A.R., Ehteshami H., 2011, Accurate solution for free vibration analysis of functionally graded thick rectangular plates resting on elastic foundation, Composite Structures, 93, 1842-1853

11. Karimi M., Haddad H.A., Shahidi A.R., 2015a, Combining surface effects and non-local two variable refined plate theories on the shear/biaxial buckling and vibration of silver nanoplates, IET Micro and Nano Letters, 10, 276-281

12. Karimi M., Mirdamadi H.R., Shahidi A.R., 2015b, Shear vibration and buckling of doublelayer orthotropic nanoplates based on RPT resting on elastic foundations by DQM including surface effects, Microsystem Technologies, 23, 1-33

13. Karimi M., Shokrani M.H., Shahidi A.R., 2015c, Size-dependent free vibration analysis of rectangular nanoplates with the consideration of surface effects using finite difference method, Journal of Applied and Computational Mechanics, 1, 122-133

14. KE L.L., WANG Y.S., 2014, Free vibration of size-dependent magneto-electro-elastic nanobeams based on the nonlocal theory, Acta Mechanical Sinica, 63, 52-61

15. Khan K., Patel B.P., Nath Y., 2014, Free and forced vibration characteristics of bimodular composite laminated circular cylindrical shells, Composite Structures, 126, 386-397

16. LANG Z., Xuewu L., 2013, Buckling and vibration analysis of functionally graded magneto-electrothermo-elastic circular cylindrical shells, Applied Mathematical Modelling, 37, 2279-2292

17. Malekzadeh P., Shojaee M., 2013, A two-variable first-order shear deformation theory coupled with surface and nonlocal effects for free vibration of nanoplates, Journal of Vibration and Control, doi: $10.1177 / / 1077546313516667$

18. Mohammadimehr M., Mohammadi-Dehabadi A.A., Khoddami Maraghi Z., 2017, The effect of non-local higher order stress to predict the nonlinear vibration behavior of carbon nanotube conveying viscous nanoflow, Physica B: Condensed Matter, 510, 48-59

19. Mohammadimehr M., Mostafavifar M., 2017, Free vibration analysis of sandwich plate with a transversely flexible core and FG-CNTs reinforced nanocomposite face sheets subjected to magnetic field and temperature-dependent material properties using SGT, Composites Part B: Engineering, 94, 253-270

20. Mohammadimehr M., Rousta Navi B., Ghorbanpour Arani A., 2016a, Modified strain gradient Reddy rectangular plate model for biaxial buckling and bending analysis of double-coupled piezoelectric polymeric nanocomposite reinforced by FG-SWNT, Composites Part B: Engineering, 87, $132-148$

21. Mohammadimenr M., Salemi M., Rousta Navi B., 2016b, Bending, buckling, and free vibration analysis of MSGT microcomposite Reddy plate reinforced by FG-SWCNTs with temperaturedependent material properties under hydro-thermo-mechanical loadings using DQM, Composite Structures, 138, 361-380 
22. Nguyen T., Nguyen V., Chau-Dinh T., Vo T., Nguyen X., 2016, Static and vibration analysis of isotropic and functionally graded sandwich plates using an edge-based MITC3 finite elements, Composites Part B, 107, 162-173

23. Nguyen-Xuan H., Rabczuk T., Bordas S., Debongnie J., 2008, A smoothed finite element method for plate analysis, Computer Methods in Applied Mechanics and Engineering, 197, 1184-1203

24. Razavi S., Shooshtari A., 2015, Nonlinear free vibration of magneto-electro-elastic rectangular plates, Composite Structures, 119, 377-384

25. Shokrani M.H., Karimi M., Tehrani M.S., Mirdamadi H.R., 2016, Buckling analysis of double-orthotropic nanoplates embedded in elastic media based on non-local two-variable refined plate theory using the GDQ method, Journal Brazilian Society of Mechanical Sciences and Engineering, 38, 2589-2606

26. Shooshtari A., Razavi S., 2015, Linear and nonlinear free vibration of a multilayered magnetoelectro-elastic doubly curved shell on elastic foundation, Composite Part B: Engineering, 78, 95-108

27. SiH G.C., Song Z.F., 2002, Damage analysis of tetragonal perovskite structure ceramics implicated by asymptotic field solutions and boundary conditions, Theoretical and Applied Fracture Mechanics, 38, 15-36

28. SiH G.C., YU H.Y., 2005, Volume fraction effect of magnetoelectroelastic composite on enhancement and impediment of crack growth, Composite Structures, 68, 1-11

29. Sовну M., 2013, Buckling and free vibration of exponentially graded sandwich plates resting on elastic foundations under various boundary conditions, Composite Structures, 99, 76-87

30. Song Z.F., Siн G.C., 2002, Electromechanical influence of crack velocity at bifurcation for poled ferroelectric materials, Theoretical and Applied Fracture Mechanics, 38, 121-139

31. Taylor R.L., Auricchio F., 1993, Linked interpolation for Reissner-Mindlin plate elements: Part II - A simple triangle, International Journal for Numerical Methods in Engineering, 36, 3057-3066

32. WAng K.F., WAng B.L., 2011, Combining effects of surface energy and non-local elasticity on the buckling of nanoplates, Micro and Nano Letters, 6, 941-943

33. Wu C.P. LU Y.C., 2009, A modified Pagano method for the 3D dynamic responses of functionally graded magneto-electro-elastic plates, Composite Structures, 90, 363-372

34. Xin L., Hu Z., 2015, Free vibration of simply supported and multilayered magneto-electro-elastic plates, Composite Structures, 121, 344-355

35. Zidour M., Daouadji T.H., Benrahou K.H., Tounsi A., Adda Bedia E.A., Hadji L., 2014, Buckling analysis of chiral single-walled carbon nanotubes by using the nonlocal Timoshenko beam theory, Mechanics of Composite Materials, 50, 95-104 